\title{
First-line targeted therapies in the treatment of metastatic colorectal cancer - role of cetuximab
}

\author{
Giuseppe Tonini \\ Alice Calvieri \\ Bruno Vincenzi \\ Daniele Santini \\ Medical Oncology, University Campus \\ Bio-Medico, Rome, Italy
}

\begin{abstract}
Worldwide, colorectal cancer (CRC) is the fourth most commonly diagnosed malignant disease and the second leading cause of cancer-related death in Western nations. In 2008 there were an estimated 148,810 new cases and 49,960 deaths in the US. For several years different chemotherapeutic regimens, based on fluoropyrimidines, irinotecan and oxaliplatin, have been used in advanced CRC, but survival is still unsatisfactory. New targeted therapies, including drugs and monoclonal antibodies (MoABs), show great promise in the fight against CRC and have shown activity in different disease settings. Cetuximab, a chimeric IgG1 monoclonal antibody that binds to the extracellular domain of epidermal growth factor receptor (EGFR), is active in metastatic colorectal cancer (mCRC). As an IgG1 antibody, cetuximab may exert its antitumor efficacy through both EGFR antagonism and antibody-dependent cell-mediated cytotoxicity. The combination of this drug with classical chemotherapies has shown better clinical profiles reflected in an improvement in overall and progression-free survival. Clinical trials established the role of cetuximab, particularly with irinotecan, in irinotecan-refractory/heavily pretreated patients. Whereas cetuximab has a clear indication in the salvage setting, its role in first-line therapy remains investigational. It is particularly encouraging that cetuximab may enhance curative opportunities in patients with early metastatic disease, suggesting that adding cetuximab in first-line therapy may downstage disease in some patients, and, as a result, allow potentially curative resection of previously unresectable metastases. In this review we will focus on the main epidermal growth factor receptor inhibitors demonstrating clinical benefit, and the role of cetuximab in first-line treatment of metastatic CRC.
\end{abstract}

Keywords: cetuximab, colorectal cancer, clinical trials

\section{Introduction}

In Europe, colorectal cancer (CRC) was estimated to be the second most frequent cause of cancer-related death in 2006. ${ }^{1}$ Although there have been substantial advances in the treatment of metastatic CRC (mCRC), median survival remains less than 2 years and less than $5 \%$ of patients survive for more than 5 years. ${ }^{2,3,4,5,6,7}$

Chemotherapy (CT) is the mainstay of the treatment of $\mathrm{mCRC}$, and, except for a minority of patients who are candidates for salvage surgery, in most cases its goal is palliation. The standard chemotherapy for patients with advanced CRC (ACC) consists of fluoropyrimidines, irinotecan and oxaliplatin. ${ }^{8}$ Since the introduction of 5-fluorouracil (5-FU), CT has proved to prolong median survival time (MST) of patients with advanced disease. With optimal scheduling, 5-FU doubled MST compared with best supportive care alone. ${ }^{9,10}$ Recent advances in combination $\mathrm{CT}$, by addition of either oxaliplatin or irinotecan to 5-FU, resulted in improvement in both response rate (RR) and overall survival (OS), ${ }^{11}$ with maximal benefit obtained when patients receive all three agents during the course of their disease. ${ }^{12}$ Moreover, combination CT has improved the possibility of downstaging metastatic patients, thereby making resectable an additional $12 \%$ to $20 \%$ of patients initially considered unresectable. ${ }^{13}$ When these drugs are made available to patients during the course of their disease, a median survival of 17 to 20 months may be achieved. \footnotetext{
University Campus Bio-Medico, Rome, Medical Oncology,Via Alvaro del Portillo 200, 00128 Rome, Italy Email g.tonini@unicampus.it
}

Correspondence: Giuseppe Tonin 
The treatment of ACC has made considerable progress in recent years. The newest developments are monoclonal antibodies (MoABs ) such as cetuximab, which have shown activity in different disease settings. ${ }^{14,15}$ The epidermal growth factor receptor (EGFR) is a $170-\mathrm{kDa}$ transmembrane glycoprotein, which is a member of the ErbB family of receptors. Ten different ligands can selectively bind to each receptor. After a ligand binds to a single-chain EGFR, the receptor forms a dimer ${ }^{16}$ that signals within the cell by activating receptor autophosphorylation through tyrosine kinase activity. ${ }^{17}$ Autophosphorylation triggers a series of intracellular pathways that may result in cancer cell proliferation, differentiation, migration, and adhesion, and enhances critical tumor growth and progression, including angiogenesis, apoptosis inhibition, tumor invasiveness, and metastatic spread (Figure 1).

\section{Mechanisms of action of anti-EGFR drugs in cancer cells}

The first anti-EGFR drugs were been developed in the 1980s. ${ }^{18}$ Anti-EGFR monoclonal antibodies such as cetuximab bind to the EGFR extracellular domain during the inactive configuration. The occlusion of the ligand-binding region prevents the interaction between the receptor and the ligand, inibiting ligand-induced EGFR tyrosine kinase activation. ${ }^{19,20}$

Cetuximab, an IgG1 monoclonal antibody, specifically targets EGFR with a higher affinity than its natural ligands. ${ }^{21}$ Cetuximab prevents EGFR from binding to these endogenous ligands and therefore from adopting the extended configuration necessary for dimerization and signal transduction. ${ }^{22}$ Monoclonal antibody binding also stimulates receptor internalization and degradation. ${ }^{23,24}$ In preclinical models, cetuximab has been shown to increase tumor cell apoptosis, ${ }^{25,26}$ suppress invasion and metastasis, ${ }^{27}$ inhibit proliferation, ${ }^{28}$ and downregulate the production of proangiogenic factors (Figure 2). ${ }^{29}$

Additional improvements in treatment are likely to be facilitated by the use of rationally selected therapeutic agents that target functionally important proteins in tumor cells, such as EGFR, expressed in $75 \%$ to $89 \%$ of CRCs. ${ }^{30,31}$ EGFR is often overexpressed in CRC and EGFR expression levels have been shown to be related to prognosis: higher EGFR

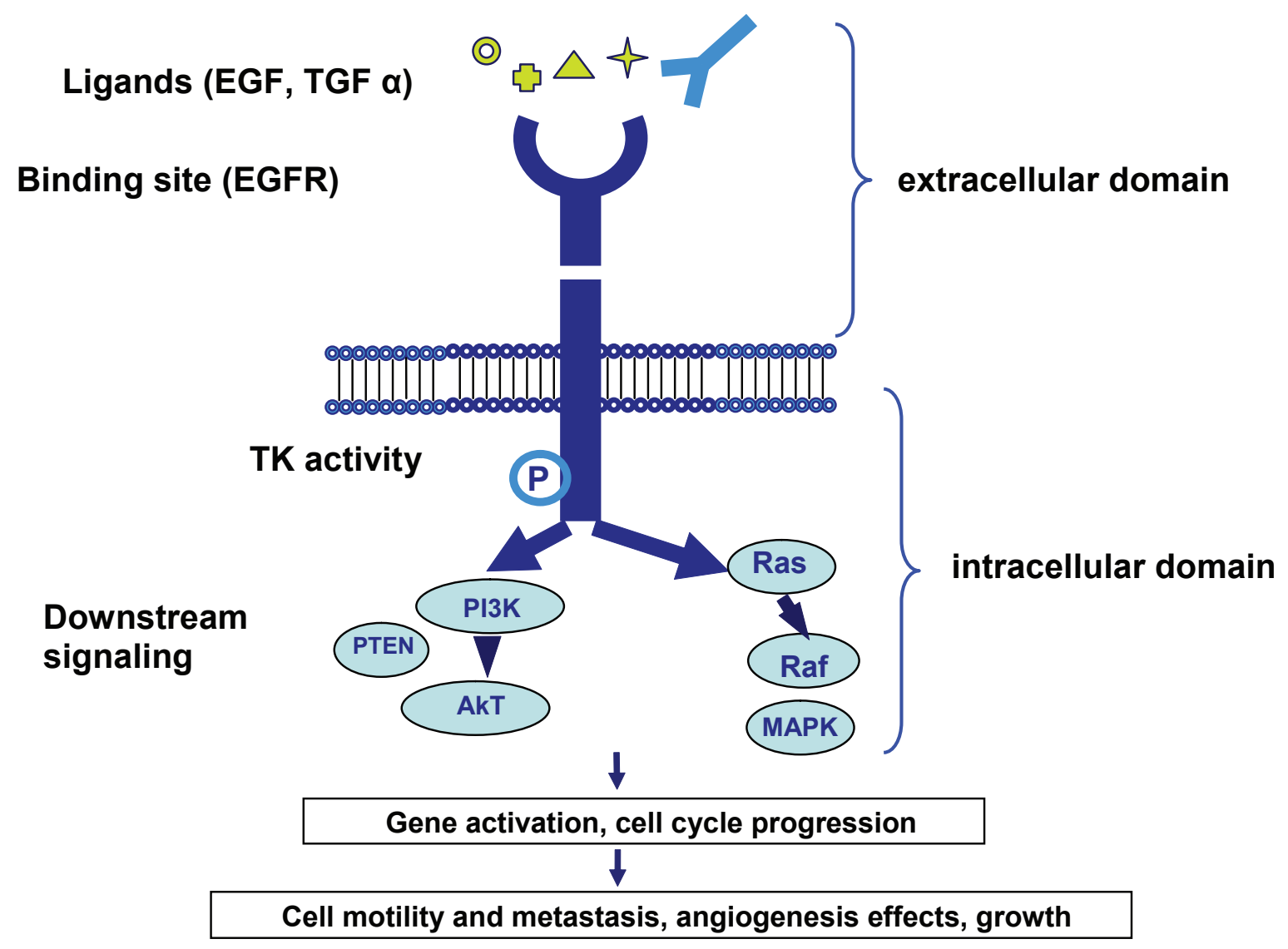

Figure I Signal transduction pathways controlled by the activation of EGFR.

a) The binding of a receptor-specific ligand occurs in the extracellular portion or of one of the EGFR-related receptors (HER2, HER3, HER4). b) The information of a functionally active EGFR-EGFR dimer causes the ATP-dependent phosphorylation of specific tyrosine residues in the EGFR intracellular domain. c) This phosphorylation triggers a complex program of intracellular signals to the cytoplasm and then to the nucleus to activate a cascade of anti-apoptotic and pro-survival signals. 


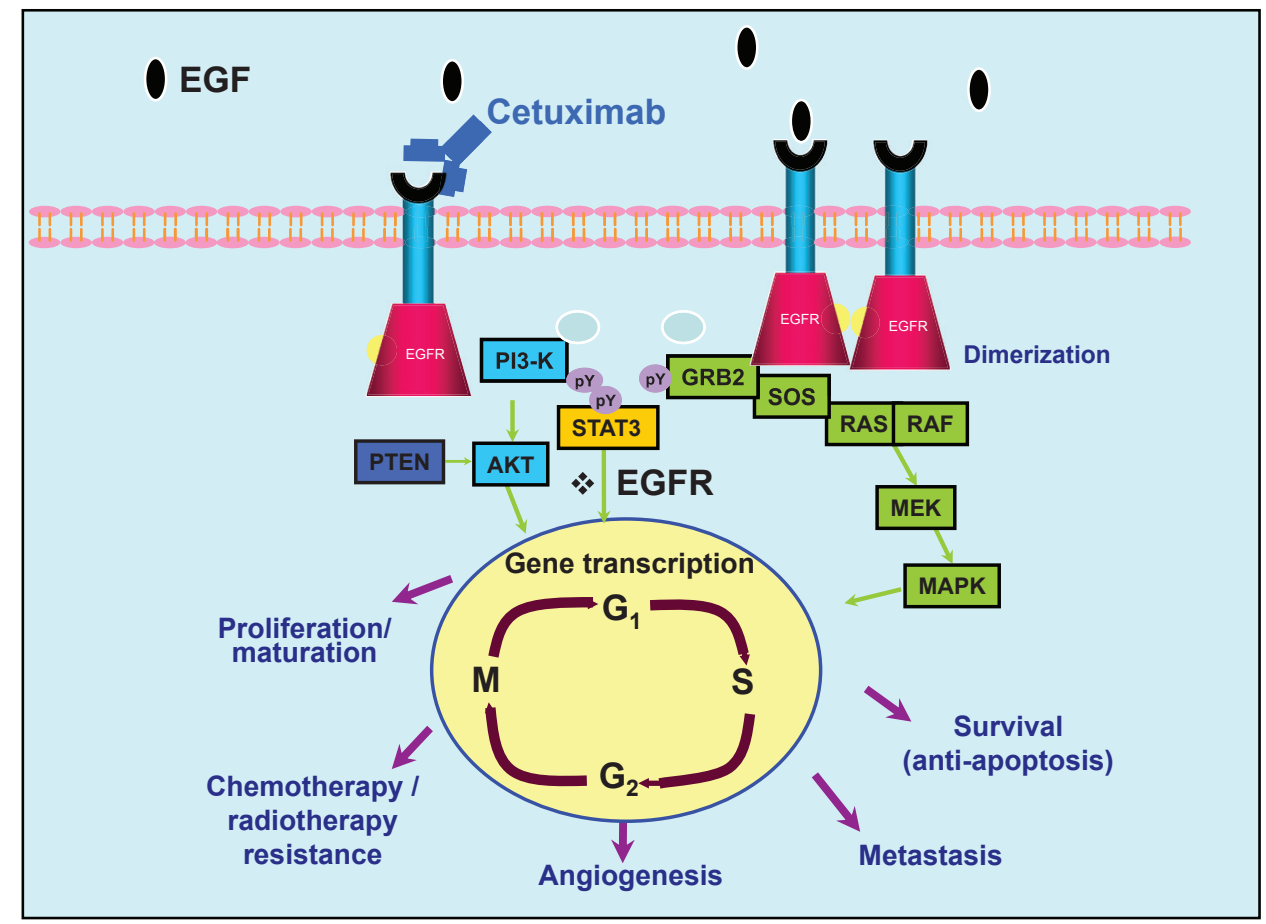

Figure 2 Monoclonal antibody cetuximab blocks EGFR. The anti-EGFR monoclonal antibody cetuximab, which is an lgGI immunoglobulin, could elicit host antitumor immune responses, including antibody-dependent, cell-mediated cytotoxicity and can induce EGFR cellular internalization and downregulation, thereby enhancing receptor degradation. These two mechanisms could make an important contribution to antitumor activity.

expression levels correlating with a shorter OS and greater metastatic potential. ${ }^{32}$

\section{Cetuximab in first-line treatment of metastatic colorectal cancer Preclinical cancer models}

Experimental and clinical studies support the hipotesis that the EGFR represents a relevant target for cancer therapy. ${ }^{16}$ The possibility of combining conventional cytotoxic drugs with novel agents that specifically interfere with key pathways in controlling cancer cell survival, proliferation, invasion and/or metastatic spreading has generated a wide interest. Since the cellular targets of new agents are different from those of cytotoxic drugs, it is possible to combine them with standard chemotherapy without the risk of cross-resistance. The modification of the expression and/or the activity of genes regulating mitogenic signals can directly cause a perturbation of cell growth, but can also affect cancer cell sensitivity to conventional chemotherapy and radiotherapy. The EGFR autocrine pathway is one of the most active growth factor-driven mechanisms implied in the development and in the progression of human epithelial cancers. For this reason, in the past few years several preclinical and clinical studies have been conducted to test the antitumor activity of selective EGFR antagonists and cytotoxic therapies.
Morelli et $\mathrm{al}^{33}$ demonstrated, through an in vitro model of human cancer epithelial cell line with a functional EGFR-dependent autocrine growth pathway, that antiproliferative activity potentiation of selected cytotoxic drugs (such as platinum derivatives and taxanes) combined with EGFR inhibitors was both schedule- and sequence-dependent. This model clearly demostrated how a synergistic effect in inhibiting cancer growth could be observed only in those neoplastic cells first exposed to a cytotoxic drug and then treated with an EGFR inhibitor. Moreover, cancer cells death was significantly increased and the surviving cells tended to accumulate in G2/M phases, showing a modification in cell cycle distribution.

Many preclinical studies have been done to understand the mechanisms through which cetuximab succeeds in enhancing oxaliplatin's antiproliferative effect. In the Balin-Gauthier et $\mathrm{a}^{34}$ study, the interaction between cetuximab and oxaliplatin antitumoral activities was tested on a panel of human CRC cell lines (HCT-8, HT-29, SW620, HCT-116). It was shown that observed responses were strictly dependent on cell type and on phospho-EGFR basal level, without any significant correlation with EGFR expression level. Cetuximab was also proven to lower oxaliplatin $\mathrm{IC}_{50}$ values when tested, in vitro, with HCT-8 (EGF-R moderate) and 
HT-29 (EGF-R weak) cell lines, while no major modification was found with HCT-116 or SW620.

In a recent study Balin-Gauthier et $\mathrm{al}^{35}$ used responsive (HCT-8) and non-responsive (HCT-116) human colon cancer cell lines to analyze the effect of cetuximab on oxaliplatin intracellular detoxification, DNA adduct formation, cell cycle distribution, and induction of apoptosis. In responsive HCT-8 CRC cell line, cetuximab induces platinum-DNA adducts formation. This promotion is associated with a decreased expression of ERCC1 enzyme (excision repair cross complementation group 1) that seems to have a key role in the oxaliplatin-DNA platinum adduct repair process, the nucleotide excision repair (NER). They also observed a reduced expression of factors involved in DNA replication initiation, which correlates with an enrichment of G1 phase cells, and the stimulation of apoptosis pathway with an acceleration of cells death. None of these changes occurred in the non-responsive HCT-116 cell line, showing multiple interactions between oxaliplatin pharmacology and the EGFR signaling transduction pathway. These results suggested that under oxaliplatin treatment, the presence of adducts prevents cells from repliction, decreasing the number of $S$ phase cells. On the other hand, cetuximab combination therapy modified oxaliplatin adduct repair and induced modifications in the early stages of HCT-8 DNA.

Further studies by Prewett et $\mathrm{al}^{36}$ investigated the effects of cetuximab and oxaliplatin treatment, single-agent or in combination, in vitro and in vivo, by using human CRC cell lines selected for oxaliplatin resistance, as well as parental control cell lines. Subcutaneous xenograft tumor growth in nu/nu athymic mice was evaluated, as well as activation of mitogen-activated protein kinase (extracellular signal-regulated kinase 1/2) and AKT, expression of DNA repair genes, density of apurinic/apyrimidinic DNA damage, and accumulation of platinum-DNA adducts in vitro. Oxaliplatin and cetuximab combination efficacy in murine subcutaneous xenograft models was greater than the respective monotherapy activity without any correlation with oxaliplatin monotherapy responsiveness. In vitro, cetuximab reduced expression of excision repair cross-complementation group 1 and XPF, which are key components of the nucleotide excision repair pathway involved in the cutting of platinumDNA adducts. In addition, cetuximab reduced expression of XRCC1, a component of the base excision repair pathway responsible for repairing apurinic/apyrimidinic sites. Effects of cetuximab on DNA reparation proteins were downstream to the effects on mitogen-activated protein kinase and AKT pathway activation. In line with effects on DNA repair protein expression, cetuximab increased the accumulation of platinum and apurinic/apyrimidinic sites on DNA during oxaliplatin treatment.

In summary, these preclinical studies illustrate cetuximab activity in the restoration of chemosensitivity to cytotoxic agents and provide a rationale for the evaluation of a potentially synergistic sequence of cytotoxic drugs and EGFR inhibitors in a clinical setting.

\section{Clinical application}

Building upon the effectiveness of cetuximab in refractory mCRC, several studies were conduced to explore whether adding cetuximab to first-line therapy would improve patients outcome.

A single-arm phase Ib/II study was conducted by Arnold et $\mathrm{al}^{37}$ in order to investigate the feasibility of administering cetuximab in combination with oxaliplatin and infusional 5-fluorouracil (5-FU)/folinic acid (FA) in a weekly schedule (Association of Internal Oncology FUFOX protocol) as first-line treatment in patients with EGFR-positive advanced CRC. Cetuximab was administered weekly: $400 \mathrm{mg} / \mathrm{m}^{2}$ initial dose, then $250 \mathrm{mg} / \mathrm{m}^{2}$, and FUFOX: oxaliplatin $50 \mathrm{mg} / \mathrm{m}^{2}$, FA $500 \mathrm{mg} / \mathrm{m}^{2}$ and 5-FU as a 24-h infusion at either 1500 or $2000 \mathrm{mg} / \mathrm{m}^{2}$ administered for 4 weeks followed by a 1 -week rest (one cycle). Dose-limiting toxicity (grade 3 diarrhea) occurred in 3 of 14 assessabled patients receiving $5-\mathrm{FU}$ at standard $2000 \mathrm{mg} / \mathrm{m}^{2}$. Cetuximab combined with FUFOX was generally well tolerated with the most common adverse events being diarrhea (27\%) and paresthesia (16\%) grade $3 / 4$. The confirmed response rate in patients receiving 5 -FU at standard $2000 \mathrm{mg} / \mathrm{m}^{2}(\mathrm{~N}=41)$ was $56 \%$, with a median response time of 9.3 months. Median progressionfree survival (PFS) and OS were, respectively, 8.1 (95\% confidence interval [CI] 6.0-9.7) and 28.2 months, including all 49 patients. Cetuximab pharmacokinetics did not seem to differ in the FUFOX combination compared with cetuximab/ irinotecan combinations.

The ACROBAT study, investigating cetuximab combined with FOLFOX-4 in 43 previously untreated ACC patients, reported similar grade 3-4 AEs, with diarrhea in $26 \%$ and paresthesia in $19 \%$ of patients, but a higher rate of neutropenia ( $23 \%$, probably due to 5 -FU bolus administration in FOLFOX-4 regimen) and rash (16\%). The results showed a $77 \%$ RR, a median survival of 30 months and a median PFS of 12.3 months. Twenty-three percent of these patients had their liver metastases resected subsequently. ${ }^{38}$ The favorable results of the ACROBAT trial are due merely 
to selection of patients with favorable prognostic factors for cetuximab-based combination chemotherapy. In addition to clinical factors (eg, smaller metastases load), this could be related to molecular prognostic or predictive factors.

The study of Rosemberg et al in $2002^{39}$ was designed as a phase II study enrolling 27 EGFR-positive patients treated with irinotecan, 5-fluorouracil/leucovorin (IFL) and cetuximab as frontline. The data showed a $44 \%$ partial response (PR) rate with a $20 \%$ of patients showing minor responses. Rash occurred in 26 out of 27 patients, but only $19 \%$ were grade 3 .

A similar chemotherapeutic regimen was used in the study of Folprecht et $\mathrm{al}^{40}$ in 2005, in which 21 patients with EGFR-expressing mCRC underwent cetuximab treatment in combination with weekly irinotecan and a low or high dose of infusional 5-FU. Among recruited patients $67 \%$ obtained a RR, $29 \%$ stable disease and in $20 \%$ of the cases liver metastases were resected after treatment. High and normal doses of 5-fluorouracil/leucovorin were used: in the group of high dose $\left(2000 \mathrm{mg} / \mathrm{m}^{2}\right) 3$ out of 15 patients presented dose limiting toxicities (DLT). Median survival was 33 months, and 4 patients (19\%) underwent potentially curative surgery.

A relevant phase II study conducted by Tabernero et $\mathrm{a}^{41}$ investigated the efficacy and the safety of cetuximab combined with standard oxaliplatin-based chemotherapy (infusional fluorouracil, leucovorin, and oxaliplatin [FOLFOX-4]) in the first-line treatment of EGFR-expressing mCRC. Patients with $\mathrm{mCRC}$ received on day 1 of a 14-day cycle, cetuximab (initial dose $400 \mathrm{mg} / \mathrm{m}^{2}$ during week 1 , then $250 \mathrm{mg} / \mathrm{m}^{2}$ weekly) followed by FOLFOX-4 (oxaliplatin $85 \mathrm{mg} / \mathrm{m}^{2}$ on day 1 ; leucovorin $200 \mathrm{mg} / \mathrm{m}^{2}$ on days 1 and 2 , followed by fluorouracil $400 \mathrm{mg} / \mathrm{m}^{2}$ bolus then $600 \mathrm{mg} / \mathrm{m}^{2}$ intravenous infusion during 22 hours on days 1 and 2).

The preclinical studies confirmed the supra-additive activity of cetuximab to oxaliplatin. In the clinical phase, 43 patients were included, with a median age of 65 years (range, 43 to 78 years). RRs were $79 \%$ (unconfirmed) and $72 \%$ (confirmed), with $95 \%$ of disease control. Median PFS (mPFS) and median duration of response were respectively 12.3 and 10.8 months. Ten patients (23\%) with previously unresectable metastases underwent curative resection. After a median follow-up of 30.5 months, median OS (mOS) was 30.0 months. Cetuximab did not increase FOLFOX-4 characteristic toxicity and it was generally well tolerated.

A further study with FOLFOX-6 plus cetuximab in chemonaïve patients showed a preliminary $53 \%$ overall RR (ORR) with $3 \mathrm{CR} .{ }^{42}$ This was a phase II study with $82 \mathrm{mCRC}$ with positive or undetectable EGFR expression. Fourteen patients left the study due to toxicity and $10 \%$ of the patients had grade 4 neutropenia and $2 \%$ grade 4 sepsis (Table 1 ).

The CALGB 80203 study was designed to compare first-line FOLFIRI vs FOLFOX, both with and without cetuximab. The study was closed to accrual after a total of 238 of the planned 2,200 patients were enrolled. ${ }^{43}$ Preliminary results showed that response rates were higher in the cetuximab arms, especially when administered with FOLFOX: $60 \%$ for FOLFOX plus cetuximab; $40 \%$ for FOLFOX alone; $44 \%$ for FOLFIRI plus cetuximab; and 36\% for FOLFIRI alone. Overall, adding cetuximab significantly increased RRs compared to treatment without cetuximab (52\% vs $38 \%$; $p=0.0029)$.

A more recent phase III clinical trial, the CRYSTAL study, enrolled a total of 1220 patients with untreated EGFR-expressing mCRC and randomly assigned them to cetuximab plus FOLFIRI ( $a r m$ A ) or FOLFIRI alone (arm B) treatment. Median PFS was significantly longer for arm A compared to arm B (8.9 months [CI: 8-9.5] for group A vs 8 months [CI: 7.6-9] for group $B, P=0.036$ ). RR was also significantly increased by cetuximab ( $46.9 \%$ vs $38.7 \%, \mathrm{p}=0.005$ ) compared to FOLFIRI alone. ${ }^{44}$ The most common toxicities were neutropenia $(26.7 \%$ in group A, $23.3 \%$ in group B), diarrhea ( $15.2 \%$ and $10.5 \%$ respectively) and skin reactions ( $18.7 \%$ and $0.2 \%$ respectively). The benefit derived by patients with liver disease only is particularly interesting: the resectability rates of $\mathrm{RO}$ (radical resection) increased 3-fold with the addition of cetuximab. These findings suggest that cetuximab is active in first-line setting, and especially valuable for patients with synchronous liver disease (Table 2).

The OPUS study is a phase III clinical trial ${ }^{45}$ that randomized chemo-naïve patients to FOLFOX or FOLFOX plus cetuximab. Their primary end point was response rate and secondary goals were PFS, OS, and the R0 resection rate after metastatic surgery of curative intent. The preliminary results showed an RR of $35.7 \%$ and $45.6 \%$, respectively, with 337 patients enrolled at that time. The most common

Table I Cetuximab in front-line phase II studies

\begin{tabular}{lll}
$\begin{array}{l}\text { Cetuximab } \\
\text { + FOLFIRI }\end{array}$ & $\begin{array}{l}\text { Cetuximab } \\
\text { + FOLFOX-4 }\end{array}$ & $\begin{array}{l}\text { Cetuximab } \\
\text { + FOLFOX-6 }\end{array}$ \\
\hline 22 & 43 & 82 \\
80 & 77 & 53 \\
10.9 & 12.3 & - \\
- & 30 & - \\
\hline
\end{tabular}

Abbreviations: Pts, patients; RR, response rate; PFS, progression-free survival; OS, overall survival. 
Table 2 Clinical studies of cetuximab in first-line treatment of EGFR-expressing mCRC

\begin{tabular}{|c|c|c|c|c|c|}
\hline Study Type Phase II & $\begin{array}{l}\text { Treatment } \\
\text { (No of patients) }\end{array}$ & $\begin{array}{l}\text { ORR } \\
(C R+P R)(\%)\end{array}$ & $\begin{array}{l}\text { mTTP } \\
\text { (months) (\%) }\end{array}$ & $\begin{array}{l}\text { mPFS } \\
\text { (months) (\%) }\end{array}$ & $\begin{array}{l}\text { MOS } \\
\text { (months) (\%) }\end{array}$ \\
\hline ACROBAT trial Andrè et $\mathrm{al}^{38}$ & Cetuximab + Folfox $-4(43)$ & 77 & NR & 12.3 & 30.0 \\
\hline Folprecht et $\mathrm{a}^{31}$ & Cetuximab + Irinotecan +5 -FU/FA (2I) & 67 & 9.9 & NR & 33 \\
\hline SAKK Group Borner et al $\left.\right|^{64}$ & $\begin{array}{l}\text { Cetuximnab }+ \text { Xelox (37) } \\
\text { vs Xelox (74) }\end{array}$ & $\begin{array}{l}35.1 \\
54\end{array}$ & $\begin{array}{l}5.8 \\
7.2\end{array}$ & NR & $\begin{array}{l}16.5 \\
20.5\end{array}$ \\
\hline Study Type phase III & Treatment (no. of patients) & $\begin{array}{l}\text { ORR } \\
(C R+P R)(\%)\end{array}$ & $\begin{array}{l}\text { mTTP } \\
\text { (months) (\%) }\end{array}$ & $\begin{array}{l}\text { mPFS } \\
\text { (months) (\%) }\end{array}$ & $\begin{array}{l}\text { MOS } \\
\text { (months) (\%) }\end{array}$ \\
\hline OPUS trial (on going) 45 & $\begin{array}{l}\text { Folfox vs } \\
\text { Folfox + Cetuximab (337) }\end{array}$ & $\begin{array}{l}35.7 \\
45.6\end{array}$ & & & \\
\hline Phase III CALGB $80203^{43}$ & $\begin{array}{l}\text { Folfiri (6I) } \\
\text { Folfiri + Cetuximab (59) } \\
\text { Folfox (60) } \\
\text { Folfox + Cetuximab (58) }\end{array}$ & $\begin{array}{l}36 \\
44 \\
40 \\
60\end{array}$ & $\begin{array}{l}38 \\
32 \\
30 \\
26\end{array}$ & $\begin{array}{l}8.4 \\
10.6 \\
9.8 \\
8.2\end{array}$ & NR \\
\hline $\begin{array}{l}\text { Phase III COIN trial } \\
\text { (ongoing) }^{46}\end{array}$ & $\begin{array}{l}\text { Continuous Folfox } \\
\text { Continuous Folfox }+ \text { Cetuximab } \\
\text { CT for } 12 \text { weeks followed by its } \\
\text { interruption and resumption on PD }\end{array}$ & NR & NR & NR & NR \\
\hline Phase III CRYSTAL ${ }^{44}$ & $\begin{array}{l}\text { Folfiri }(600) \text { vs } \\
\text { Folfiri + Cetuximab }(602)\end{array}$ & $\begin{array}{l}38.7 \\
46.9 \\
(p=0.004)\end{array}$ & N.R & $\begin{array}{l}8 \\
8.9 \\
(p<0.04)\end{array}$ & NR \\
\hline
\end{tabular}

Abbreviations: CRC, colorectal cancer; ORR, overall response rate; CR, complete response; PR, partial response; mPTT, median time to progression; mPFS, median progression-free survival; mOS, median overall survival; NR, not reported.

grade 3-4 adverse events were neutropenia $(27.6 \%$ in $\mathrm{A} ; 31.5 \%$ in B), diarrhea (7.1\% and 6.0\%), leucopenia (7.1\% and $5.4 \%)$ and rash (9.4\% in the cetuximab arm only).

The ongoing UK Medical Research Council's COIN phase III trial ${ }^{46}$ in $\mathrm{mCRC}$ is going to randomize approximately 2,400 first-line patients, whose EGFR status has not been previously estabilished, to one of three arms: continuous oxaliplatin and fluoropyrimidine chemotherapy, continuous chemotherapy plus cetuximab, or chemotherapy for 12 weeks followed by its interruption and resumption on disease progression. The addition of cetuximab to oxaliplatin/fluoropyrimidine combinations results in increased grade 3-4 toxicities, and gastrointestinal (GI), skin rash and lethargy seem to be the most represented.

The results of these ongoing studies will elucidate the role of these combination in first-line treatment.

\section{Cetuximab in combination with other novel agents}

Animal models suggest that additive efficacy and sometimes synergy can be achieved using EGFR inhibitors in combination with agents that inhibit the vascular endothelial growth factor receptor. ${ }^{47,48}$ The EGFR pathway rules the production of vascular endothelial growth factor (and other angiogenic factors); targeting both markers may therefore have a greater antitumor effect.

The addition of bevacizumab (a humanized mAb against the vascular endothelial growth factor) to fluoropyrimidine-containing chemotherapy ${ }^{49}$ results in a significant survival benefit and it is currently considered as part of standard first-line therapy. In irinotecan monotherapy-resistant patients, the use of cetuximab improves mPFS. Cetuximab monotherapy improves the mOS in ACC patients in whom all available standard treatments have failed. ${ }^{50}$ In ACC first-line treatment, cetuximab improves PFS when given in combination with bolus 5-FU plus FOLFIRI compared with FOLFIRI alone, although the absolute increase in mPFS is modest ( 8.9 vs 8.0 months). So far, limited data are available on the efficacy and safety of the combination of bevacizumab and anti-EGFR targeted agents in ACC. A randomized phase II study comparing bevacizumab, cetuximab and irinotecan with bevacizumab and cetuximab in irinotecan-resistant patients with ACC (BOND-2) showed an acceptable toxicity profile in both arms and a median time to progression of 7.9 vs 5.6 months. ${ }^{51}$

The Cancer and Leukemia Group B (CALGB/SWOG 80405 ) plans to randomize 2,289 $\mathrm{mCRC}$ patients for a firstline treatment with chemotherapy (FOLFOX or FOLFIRI) plus bevacizumab or cetuximab vs the combination of bevacizumab plus cetuximab. Patients and their 
physicians will first choose either the FOLFOX or FOLFIRI chemotherapy regimen and then will be randomized to treatment with cetuximab, bevacizumab or both. The primary endpoint is OS, with RR, PFS, duration of response, and time to progression as secondary endpoints. Additionally, this study will explore witch regimen is the most likely to allow patients to undergo metastases potentially curative surgery after chemotherapy. ${ }^{52}$

CAIRO2 is a randomized phase III trial, evaluating the possibility of adding cetuximab to a regimen of capecitabine, oxaliplatin, and bevacizumab in order to improve PFS in previously untreated mCRC patient. Secondary end points are OS, tumor response, duration of response, quality of life, safety and translational research on factors that predict the response to treatment. 750 patients will be enrolled. Treatment consistes of capecitabine $1000 \mathrm{mg} / \mathrm{m}^{2}$ given orally twice daily on days $1-14$, oxaliplatin $130 \mathrm{mg} / \mathrm{m}^{2}$ iv on day 1 and bevacizumab $7.5 \mathrm{mg} / \mathrm{kg}$ iv on day 1 (arm A) or the same schedule plus cetuximab $400 \mathrm{mg} / \mathrm{m}^{2}$ iv in week 1 of the first treatment cycle and $250 \mathrm{mg} / \mathrm{m}^{2}$ iv weekly thereafter (arm B). All cycles are administered every 3 weeks. The study is still ongoing, but preliminary results on an interim analysis of toxicity show that the incidence of overall grade 3-4 toxicity is significantly higher in arm B compared with arm A (81\% vs $72 \%, \mathrm{p}=0.03)$. This difference is fully attributed to cetuximab-related skin toxicity. The addition of cetuximab does not seem to result in an increase of gastrointestinal toxicity or treatment-related mortality. ${ }^{53}$

\section{Response to anti-EGFR drugs: clinical and pathological predictors}

Since only a subgroup of patients with cancer have a clinical benefit from treatment with EGFR inhibitors, there is an urgent need for identification and clinical validation of useful criteria in selecting patients for such treatment.

A cetuximab-peculiar toxic effect is a papulopustural skin rash, generally on the face and upper torso, which is thought to be mechanism- and dose-related. ${ }^{54}$ Findings suggest that there is a correlation between intensity of skin rash, response and survival. ${ }^{55}$ This correlation is particularly striking in a subgroup analysis from the IMC 0144 trial reported by Pippas et al. In that trial, patients with no skin toxicity presented no objective responses and had a median survival of 1.7 months, whereas those who experienced grade 3 skin rash had a $20 \%$ $\mathrm{RR}$ and a mOS of almost 1 year. ${ }^{56}$ This is the first reported observation of a clinical feature that may predict the clinical outcome of an antitumor agent. Dose-escalation schedules are currently under investigation in order to explore the possibility of increasing cetuximab efficacy by inducing skin rash.

However, the presence and severity of cutaneous toxic effects are the most important clinical correlates of the efficacy of anti-EGFR therapy. The skin effect reflects the extent of EGFR blockade achieved in the tumor and correlates with EGFR saturation and with relevant drug concentration within the target.

EGFR expression, determined by immuno-histochemical methods, was the first biomarker investigated as a potential predictor of response. However, most studies have failed to show any relationship between EGFR expression and the clinical activity of anti-EGFR drugs. ${ }^{57}$ Cetuximab has also been shown to have clinical activity in patients with EGFRnegative CRC. ${ }^{58}$ Collectively, these data suggest that EGFR immuno-histochemical testing is not an optimal method for identifying patients who may have a response to anti-EGFR drug treatment.

Lievre et al recently reported that tumors with $K R A S$ mutation are associated with resistance to cetuximab therapy. ${ }^{59}$ In their study, 11 out 30 patients (37\%) responded to cetuximab therapy, which was mostly given in combination with irinotecan alone (75\%) and in a multi-refractory setting (80\%). KRAS mutation was detected in 13 of the 19 nonresponder patients, but none of the responders was found to be positive $(\mathrm{p}=0.0003)$.

$K R A S$ activating mutation, which results in an increase in the mitogen-activated protein kinase pathway, is detected in approximately $40 \%$ to $45 \%$ of patients with CRC, and its presence seems to be correlated with a worse prognosis. $K R A S$ mutations are frequently recorded codons 12 and 13 of exon 2 and usually eliminate the possibility of a concurrent EGFR mutation.

In several studies, $K R A S$ mutations have been associated with lack of response to cetuximab in patient with advanced chemotherapy-refractory colorectal cancer. ${ }^{60,61}$ These studies suggest that constitutive activation of the KRAS signaling pathway could impair the response to anti-EGFR drugs. ${ }^{62}$

Bokemeyer et al ${ }^{63}$ evaluated the influence of $K R A S$ mutation in patients treated with standard first-line therapy, whether or not associated with cetuximab. Best OR and PFS time (IRC evaluation) are linked to $K R A S$ mutation presence. $K R A S$ mutations were detected in $42 \%(99 / 233)$ of evaluable samples. Their data suggest that the benefit from addition of cetuximab to standard treatment is higher for the population with wild-type $K R A S$. For patients with $K R A S$ mutations, no major benefit has been shown from adding cetuximab to FOLFOX regimen (Table 3). 
Table 3 Clinical studies of cetuximab in combination with other novel agent in first-line treatment

\begin{tabular}{llll}
\hline Study type & Treatment & Patients & MOS (months) (\%) \\
\hline Randomized phase II study & Cetuximab + Bevacizumab + Irinotecan & 43 & 14.5 \\
(BOND-2) Saltz et al ${ }^{51}$ & vs Cetuximab + Bevacizumab & 40 & 11.4 \\
Randomized phase III study & Folfox/Folfiri + Bevacizumab or Cetuximab & Plans to randomize 2,289 patients & Still ongoing \\
$(\text { CALGB/ SWOG 80405) })^{52}$ & vs Folfox/ Folfiri + Bevacizumab + Cetuximab & & Still ongoing \\
Randomized phase III study & Capecitabine + Oxaliplatin + Bevacizumab & Plans to randomize 750 patients \\
${\text { (CAIRO2) })^{53}}^{\text {vs Capecitabine + Oxaliplatin + Bevacizumab + }}$ & & \\
& Cetuximab & & \\
\hline
\end{tabular}

Abbreviation: MOS, median overall survival.

These data suggest the possibility of choosing the most suitable treatment (cetuximab vs bevacizumab) based on KRAS analysis.

\section{Conclusions}

The results from the studies reviewed clearly point out the major role played by cetuximab in advanced CRC. Cetuximab has been used successfully in patients with refractory mCRC, and nowadays its role in the management of this tumor is growing. A defined role in the treatment of mCRC was established for regimens based on cetuximab plus irinotecan. Encouraging activity has also been observed in first-line treatment in combination with FOLFIRI and FOLFOX. Data from phase II trials in metastatic disease seem to indicate better results obtained with the addition of cetuximab to standard chemotherapy treatments (in particular, to oxaliplatincontaining regimens). On the basis of available evidence, the best interaction seems to be achieved when cetuximab is combined with oxaliplatin in first-line treatment and with irinotecan in refractory disease. The reason for these results is still unclear, but they suggest that interactions between cetuximab and oxaliplatin involve pathways different from those between cetuximab and irinotecan. Pending data from phase III trials will clarify their role in this setting.

It is also particularly evident that cetuximab may enhance the curative opportunities in patients with early metastatic disease. The early use of cetuximab combined with cytotoxic therapy during a treatment course could perhaps make a higher fraction of mCRC fully resectable, as asserted by the significantly higher R0 resection rate reported for the cetuximab plus fluorouracil, leucovorin, and irinotecan arm of the Cetuximab Combined With Irinotecan in First-Line Therapy for Metastatic Colorectal Cancer (CRYSTAL) study. Although most of the patients (91\%) experienced grade 3-4 adverse events, the combination of cetuximab and FOLFOX-4 was generally well tolerated, and there was no evidence to suggest that cetuximab increased the frequency or the severity of usual FOLFOX toxicities.

Preliminary evidence suggests that adding cetuximab to first-line therapy may downstage disease in metastatic patients, allowing a potentially curative resection of previously unresectable lesions. The significant improvement in resection rate may increase OS.

Future utilization of cetuximab in mCRC treatment could combine the activity of the two different biologic agents: cetuximab and bevacizumab. The combination of cetuximab and bevacizumab, alone or together with other standard drugs, appears tolerable and active, and warrants investigation as front-line combination chemotherapy regimens. It is likely that some patients may benefit more from FOLFOX plus bevacizumab, others from FOLFIRI plus cetuximab, and still others from different combination of chemotherapy and biologic agents. The key will be to determine predictive factors, which will help us to identify the treatment that best suits the patient. The observation that KRAS gene mutation is associated with resistance to cetuximab is a promising first step.

Table 4 Median progression-free survival (mPFS) and overall response rate (RR) by KRAS mutation status

\begin{tabular}{|c|c|c|c|c|c|}
\hline \multirow{2}{*}{$\begin{array}{l}\text { KRAS status } \\
\text { Wild-type }\end{array}$} & \multirow{2}{*}{$\begin{array}{l}\text { Median PFS }(\mathbf{m o}) \\
\text { Cetuximab + FOLFOX } \\
7.7(n=61)\end{array}$} & \multicolumn{2}{|c|}{$\begin{array}{l}\text { Median PFS (mo) } \\
\text { FOLFOX }\end{array}$} & \multirow{2}{*}{$\begin{array}{l}\text { Overall RR }(\%) \\
\text { Cetuximab + FOLFOX } \\
6 I(n=6 I)\end{array}$} & \multirow{2}{*}{$\begin{array}{l}\begin{array}{l}\text { Overall RR (\%) } \\
\text { FOLFOX }\end{array} \\
37(n=73) p=0.01\end{array}$} \\
\hline & & $7.2(n=73)$ & HR: $0.57 p=0.02$ & & \\
\hline Mutation & $5.5(n=52)$ & $8.6(n=47)$ & HR: $1.83 p=0.02$ & $33(n=52)$ & $49(n=47) p=0.11$ \\
\hline
\end{tabular}

Abbreviation: HR, hazard ratio. 
All these data must encourage clinicians and basic researchers to maintain their efforts to untangle the EGFR network, in order to improve patient quality of life and survival.

\section{Disclosures}

None of the authors have conflicts of interest to disclose.

\section{References}

1. Ferlay J, Autier P, Boniol M, et al. Estimates of the cancer incidence and mortality in Europe in 2006. Ann Oncol. 2007;18:581-592.

2. Douillard JY, Cunningham D, Roth $\mathrm{AD}$, et al. Irinotecan combined with fluorouracil compared with fluorouracil alone as first-line treatment for metastatic colorectal cancer: A multicentre randomised trial. Lancet. 2000;355:1041-1047.

3. Saltz LB, Cox JV, Blanke C, et al. Irinotecan plus fluorouracil and leucovorin for metastatic colorectal cancer: Irinotecan Study Group. N Engl J Med. 2000;343:905-914.

4. Köhne CH, van Cutsem E, Wils J, et al. Phase III study of weekly highdose infusional fluorouracil plus folinic acid with or without irinotecan in patients with metastatic colorectal cancer: European Organisation for Research and Treatment of Cancer Gastrointestinal Group Study 40986. J Clin Oncol.2005;23:4856-4865.

5. De Gramont A, Figer A, Seymour M, et al. Leucovorin and fluorouracil with or without oxaliplatin as first-line treatment in advanced colorectal cancer. J Clin Oncol. 2000;18:2938-2947.

6. Giacchetti S, Perpoint B, Zidani R, et al. Phase III multicenter randomized trial of oxaliplatin added to chronomodulated fluorouracil-leucovorin as first-line treatment of metastatic colorectal cancer. J Clin Oncol. 2000;18:136-147.

7. Grothey A, Deschler B, Kroening H, et al. Phase III study of bolus 5-fluorouracil (5-FU)/ folinic acid (FA) (Mayo) vs weekly high-dose $24 \mathrm{~h}$ 5-FU infusion/ FA + oxaliplatin (OXA) (FUFOX) in advanced colorectal cancer (ACRC). Proc Am Soc Clin Oncol. 2002;21:129a. (abstr 512).

8. Punt CJ. New options and old dilemmas in the treatment of patient with advanced colorectal cancer. Ann Oncol. 2004;15(10):1453-1459.

9. Petrelli N, Douglas HO Jr, Herrera L, et al. The modulation of fluorouracil by leucovorin in metastatic colorectal carcinoma: a prospective randomized phase III trial. Gastrointestinal Tumor Study Group. J Clin Oncol. 1989;7:1419-1426.

10. De Gramont A, Bosset JF, Milan C, et al. Randomized trial comparing monthly low dose leucovorin and fluorouracil bolus with bimonthly high dose leucovorin and fluorouracil bolus plus continuous infusion for advanced colorectal cancer: a French Intergroup Study. J Clin Oncol. 1977;15:808-815.

11. Kelly H, Goldberg RM, et al. Systemic therapy for metastatic colorectal cancer: current options, current evidence. J Clin Oncol. 2005;23:4553-4560

12. Grothey A, Sargent D, Goldberg RM, et al. Survival of patients with advanced colorectal cancer improves with the availability of fluorouracil-leucovorin, irinotecan and oxaliplatin in the course of treatment. J Clin Oncol. 2004;22:1209-1214.

13. Tournigand $\mathrm{C}$, Andre T, Achille E, et al. FOLFIRI followed by FOLFOX6 or the reverse sequence in advanced colorectal cancer: a randomized GERCOR study. J Clin Oncol. 2004;22:229-237.

14. Cunningham D, Humblet $Y$, Siena $S$, et al. Cetuximab monotherapy and cetuximab plus irinotecan in irinotecan-refractory metastatic colorectal cancer. N Engl J Med. 2004;351:337-345.

15. Saltz LB, Meropol NJ, Loehrer PJ Sr, et al. Phase II trial of cetuximab in patients with refractory colorectal cancer that expresses the epidermal growth factor receptor. J Clin Oncol. 2004;22: 1201-1208.
16. Salomon DS, Brandt R, Ciardiello F, et al. Epidermal growth factorreleted peptides and their receptors in human malignancies. Crit Rev Oncol Hematol. 1995;19:183-232.

17. Citri A, Yarden Y, et al. EGF-ERBB signalling: towards the systems level. Nat Rev Mol Cel Biol. 2006;7:505-516.

18. Masui H, Kawamoto T, Sato JD, et al. Growth inhibition of human tumor cells in athymic mice by anti-epidermal growth factor receotor monoclonal antibodies. Cancer Res. 1984;44:1002-7.

19. Normanno N, Bianco C, De Luca A, et al. Targe-based agents against ErbB receptors and their ligands: a novel approach to cancer treatment. Endocr Relat Cancer. 2003;10: 1-21.

20. Li S, Schmitz KR, Jeffrey PD, et al. Structural basis for inhibition of the epidermal growth factor receptor by cetuximab. Cancer Cell. 2005;7:301-311.

21. Goldstein NI, Prewett M, Zuklys K, et al. Biological efficacy of a chimeric antibody to the epidermal growth factor receptor in a human tumor xenograft model. Clin Cancer Res. 1995;1:1311-1318.

22. Li S, Schmitz KR, Jeffrey PD, et al. Structural basis for inhibition of the epidermal growth factor receptor by cetuximab. Cancer Cell. 2005;7:301-311.

23. Hadari YR, Doody JF, Wang YF, et al. 2004. The IgG1 monoclonal antibody cetuximab induces degradation of the epidermal growth factor receptor. ASCO Gastrointestinal Cancers Symposium, January 22-24, San Francisco, CA (abstract 234).

24. Prewett M, Rockwell P, Rockwell RF, et al. The biologic effects of $\mathrm{C} 225$, a chimeric monoclonal antibody to the EGFR, on human prostate carcinoma. J Immunother Emphasis Tumor Immunol. 1996; 19:419-427.

25. Overholser JP, Prewett MC, Hooper AT, et al. Epidermal growth factor receptor blockade by antibody IMC-C225 inhibits growth of a human pancreatic carcinoma xenograft in nude mice. Cancer. 2000;89:74-82.

26. Wu X, Fan Z, Masui H, et al. Apoptosis induced by an anti-epidermal growth factor receptor monoclonal antibody in a human colorectal carcinoma cell line and its delay by insulin. J Clin Invest. 1995;95:1897-1905.

27. Huang SM, Li J, Harari PM. Molecular inhibition of angiogenesis and metastatic potential in human squamous cell carcinomas after epidermal growth factor receptor blockade. Mol Cancer Ther. 2002;1:507-514.

28. Wu X, Rubin M, Fan Z, et al. Involvement of p27KIP1 in G1 arrest mediated by an anti-epidermal growth factor receptor monoclonal antibody. Oncogene. 1996;12:1397-1403.

29. Perrotte $P$, Matsumoto $T$, Inoue $K$, et al. Anti-epidermal growth factor receptor antibody $\mathrm{C} 225$ inhibits angiogenesis in human transitional cell carcinoma growing orthotopically in nude mice. Clin Cancer Res. 1999;5:257-265.

30. Goldstein NS, Armin M. Epidermal growth factor receptor immunohistochemical reactivity in patients with American Joint Committee on Cancer Stage IV colon adenocarcinoma: Implications for a standardized scoring system. Cancer. 2001;92:1331-1346.

31. Folprecht G, Lutz MP, Schoffski P, et al. Cetuximab and irinotecan/5fluorouracil/folinic acid is a safe combination for the first-line treatment of patients with epidermal growth factor receptor expressing metastatic colorectal carcinoma. Ann Oncol. 2006;17:450-456.

32. Nicholson RI, Gee JM, Harper ME. EGFR and cancer prognosis. Eur J Cancer, 2001;37 suppl 4:S9-15.

33. Morelli MP, Cascone T, Troiani T, et al. Sequence-dependent antiproliferative effects of cytotoxic drugs and epidermal growth factor receptor inhibitors. Ann Oncol. 2005;16 Suppl 4:iv61-68.

34. Balin-Gauthier D, Delord JP, Rochaix P, et al. In vivo and in vitro antitumor activity of oxaliplatin in combination with cetuximab in human colorectal tumor cell lines expressing different level of EGFR. Cancer Chemother Pharmacol. 2005;57(6):709-718.

35. Balin-Gauthier D, Delord JP, Pillaire MJ, et al. Cetuximab potentiates oxaliplatin cytotoxic effect through a defect in NER and DNA replication initiation. Br J Cancer. 2008;98(1):120-128.

36. Prewett M, Deevi DS, Bassi R, et al. Tumors established with cell lines selected for oxaliplatin resistance respond to oxaliplatin if combined with cetuximab. Clin Cancer Res. 2007;13(24):7432-40. 
37. Arnold D, T. Höhler, C. Dittrich et al. Cetuximab in combination with weekly 5-fluorouracil/folinic acid and oxaliplatin (FUFOX) in untreated patients with advanced colorectal cancer: a phase Ib/II study of the AIO GI Group. Ann Oncol. 2008;19(8):1442-1449.

38. Andre T, Tabernero J, Van Cutsem E, et al. An international phase II study of cetuximab in combination with oxaliplatin/5fluorouracil $(5 \mathrm{FU}) /$ folinic acid (FOLFOX-4) in the first-line treatment of patients with metastatic colorectal cancer (CRC) expressing Epidermal Growth Factor Receptor (EGFR) Final Results. ASCO Gastrointestinal. 2007;334 (Abstract).

39. Rosenberg AH, Loehrer PJ, Needle MN, et al. Erbitux (IMC-C225) plus weekly irinotecan (CPT-11), fluorouracil (5FU) and leucovorin (LV) in colorectal cancer (CRC) that expresses the epidermal growth factor receptor (EGFr). Proc Am Soc Clin Oncol. 2002;21:536.

40. Folprecht G, Lutz MP, Seufferlein T, et al. Cetuximab and irinotecan/ 5-FU/FA (AIO) as first line treatment in metastatic colorectal cancer (mCRC): Final results and pharmacokinetic data of a phase I/IIa study. J Clin Oncol. 2005;23:3640.

41. Tabernero J, Van Custem E, Diaz-Rubio E, et al. Phase II tial of cetuximab in combination with fluorouracil, leucovorin and oxaliplatin in the first-line treatment of metastatic colorectal cancer. J Clin Oncol. 2007;25:5225-5232.

42. Dakhil S, Cosgriff T, Headley D, et al. Cetuximab + FOLFOX6 as first line therapy for metastatic colorectal cancer (An International Oncology Network study, I-03-002). J Clin Oncol. 2006;24:3557.

43. Venook A, Niedzwiecki D, Hollis D, et al. Phase III study of irinotecan/ $\%$ FU/LV ( FOLFIRI ) or oxaliplatin ( FOLFOX ) \pm cetuximab for patients (pts) with untreated metastatic adenocarcinoma of the colon or rectum ( mCRC ): CALGB 80203 preliminary results (abstract 9). J Clin Oncol. 2006;3509.

44. Van Cutsem E, Nowacki M, and Lang S. Randomized phase III study of irinotecan and 5-FU/FA with or without cetuximab in the first-line treatment of patients with metastatic colorectal cancer (mCRC): The CRYSTAL trial. J Clin Oncol. 2007;4000 (Abstract).

45. Bokemeyer C, Bondarenko I, Makhson A, et al. Cetuximab plus 5-FU/FA/oxaliplatin (FOLFOX-4) versus FOLFOX-4 in the first-line treatment of metastatic colorectal cancer (mCRC): OPUS, a randomized phase II study. J Clin Oncol. 2007;25:4035.

46. Maughan T. on behalf of the COIN Trial Management Group and Investigators. Cetuximab (C), oxaliplatin (Ox) and fluoropyrimidine (Fp): Toxicity during the first 12 weeks of treatment for the first 804 patients entered into the MRC COIN (CR10) trial. J Clin Oncol. 2007;25:4070.

47. Jung YD, Mansfield PF, Akagi M, et al. Effects of combination antivascular endothelial growth factor receptor and anti-epidermal growth factor receptor therapies on the growth of gastric cancer in a nude mouse model. Eur J Cancer. 2000;38:1133-1140.

48. Ciardiello F, Bianco R, Damiano V, et al. Antiangiogenic and antitumor activity of anti-epidermal growth factor receptor $\mathrm{C} 225$ monoclonal antibody in combination with vascular endothelial growth factor antisense oligonucleotide in human GEO colon cancer cells. Clin Cancer Res. 2000;6:3739-3747.

49. Tol J, Punt CJA. Targeted therapy in advanced colorectal cancer, an update. Targeted Oncology. 2007;2(3):165-172.
50. Jonker DJ, O'Callaghan CJ, Karapetis CS, et al. Cetuximab for the treatment of colorectal cancer. N Engl J Med. 2007;357(20):2040-2048.

51. Saltz LB, Lenz HJ, Kindler HL, et al. Randomized phase II trial of cetuximab, bevacizumab, and irinotecan compared with cetuximab and bevacizumab alone in irinotecan-refractory colorectal cancer: the BOND-2 study. J Clin Oncol. 2007;25(29):4557-4561.

52. Venook AP, Blanke CD, Goldberg RM, et al. Assessing the combination of FOLFOX or FOLFIRI with bevacizumab, cetuximab, or both in metastatic colorectal cancer. Commun Oncol. 2006a;3:593-598.

53. Tol J, Koopman M, Rodenburg CJ, et al. A randomised phase III study on capecitabine, oxaliplatin and bevacizumab with or without cetuximab in first-line advanced colorectal cancer, the CAIRO 2 study of the Dutch Colorectal Cancer Group (DCCG). An interim analysis of toxicity. Annal Oncol. 2008; 9(4):734-738.

54. Perez-Soler R, Saltz L. Cutaneous adverse effects with HER1/ EGFR-targeted agents: is there a silver lining? J Clin Oncol. 2005;23:5235-5246.

55. Cunningham D, Humblet $Y$, Siena S, et al. Cetuximab monotherapy and cetuximab plus irinotecan in irinotecan-refractory metastatic colorectal cancer. N Engl J Med. 2004;351:337-345.

56. Pippas AW, Lenz H. J, Mayer RJ, et al. Analysis of EGFR status in metastatic colorectal cancer patients treated with cetuximab monotherapy. J Clin Oncol. 2005; (Meeting Abstracts) 23:3595.

57. Saltz L, Rubin M, Hochster H, et al. Cetuximab (IMC-C225) plus irinotecan (CPT-11) is active in CPT-11 refractory colorctal cancer (CRC) that espresse epidermal growth factor receptor (EGFR). Proc Am Soc Clin Oncol. 2001;20:3A. (Abstract).

58. Chung KY, Shia J, Kemeny Ne, et al. Cetuximab shows activity in colorectal cancer patient with tumors that do not express the epidermal growth factor receotor by immunohistochemistry. J Clin Oncol. 2005;23:1803-1810.

59. Lievre A, Bachet J-B, Le Corre D, et al. KRAS mutation status is predictive of response to cetuximab therapy in colorectal cancer. Cancer Res. 2006;66:3992-3995.

60. De Roock W, De Schutter J, De Hertogh G et al. KRAS mutations preclude tumor shrinkage of colorectal cancers treated with cetuximab [abstract]. J Clin Oncol. 2007;25(18S):4132.

61. Di Fiore F, Blanchard F, Charbonnier F et al. Clinical relevance of KRAS mutation detection in metastatic colorectal cancer treated by cetuximab plus chemotherapy. Br J Cancer. 2007;96:1166-1169.

62. De Roock W, Piessevaux H, De Schutter J, et al. KRAS wildtype state predicts survival and is associated to early radiological response in metastatic colorectal cancer treated with cetuximab. Ann Oncol. 2008;19(3):508-515.

63. C. Bokemeyer, I. Bondarenko, J. T. Hartmann et al. KRAS status and efficacy of first-line treatment of patients with metastatic colorectal cancer (mCRC) with FOLFOX with or without cetuximab: The OPUS experience. J Clin Oncol. 2008;26(May 20 suppl):abstract 4000.

64. Borner M, Koeberle D, Von Moos R, et al; Swiss Group for Clinical Cancer Research(SAKK), Bern, Switzerland. Adding cetuximab to capecitabine plus oxaliplatin(XELOX) in first-line treatment of metastatic colorectal cancer: a randomized phase II trial of the Swiss Group for Clinical Cancer Research SAKK. Ann Oncol. 2008;19(7):1288-1292. 